Radiotherapy-induced lumbosacral plexopathy in cervical cancer treatment is a very rare, but extremely serious complication. The clinical course is associated with severe bilateral lower leg pain, reduced sensation, different degrees of weakness, paresis or paralysis, and sometimes also urinary or fecal incontinence. Patient quality of life becomes significantly deteriorated. Escalating neurological disorders may make self-sufficient functioning impossible. Neurological symptoms, most often irreversible, may develop at different times after irradiation, even after more than 30 years. We present a case of neurological toxicity in a patient successfully treated for cervical cancer with pelvis and para-aortic lymph node irradiation and weekly cisplatin. Neurological symptoms developed a few weeks after completion of external irradiation, were gradually escalating and resulted in complete immobilization of the woman. We underline the significance of longterm, systematic physiotherapy and pharmacological therapy which has resulted in significant improvement of motion efficiency. The literature review concerns the questions of frequency, clinical course and mechanisms of radiationinduced plexopathy.

Key words: cervical cancer, radiochemotherapy, plexus lumbosacralis, paresis, paralysis.

\section{Radiotherapy-induced lumbosacral plexopathy in a patient with cervical cancer: a case report and literature review}

\author{
Małgorzata Klimek ${ }^{1}$ Radosław Kosobucki ${ }^{1}$, Elżbieta Łuczyńska², \\ Tomasz Bieda ${ }^{1}$, Krzysztof Urbański ${ }^{1}$
}

\author{
${ }^{1}$ Gynaecology Oncology Department, Maria Skłodowska-Curie Memorial Cancer Centre \\ and Institute of Oncology, Kraków, Poland \\ 2Radiology Department, Maria Skłodowska-Curie Memorial Cancer Centre \\ and Institute of Oncology, Kraków, Poland
}

\section{Introduction}

For a number of years, radiochemotherapy has been a treatment of choice in cervical cancer patients, starting from stage IB2. One of the complications associated with cancer treatment that is extremely rare but, at the same time, provides many diagnostic and even more therapeutic problems is radiationinduced plexopathy. The occurrence of such symptoms as sensation disorders in the lower extremities, muscular weakness, paresis, paralysis, and growing pain, raise, first of all, suspicion of nervous system involvement (pelvic nerves, spinal cord) by tumor, particularly in patients irradiated for advanced cervical cancer. Excluding the presence of cancer through clinical, laboratory and imaging examinations and tests, as well as excluding the formerly existing neuropathies, allows one to diagnose the radiation-induced nature of neurological disorders. Treatment with cytostatics, most often cisplatin, should also be taken into consideration. We present a patient in whom neurological complications developed a few weeks after completion of pelvis and paraaortic lymph node irradiation combined with cisplatin chemotherapy.

\section{Case report}

A 47-year-old woman was admitted in January 2004 to the Oncological Gynecology Department, with diagnosis of FIGO IIA squamous cell carcinoma of the cervix. Chest x-ray did not show any pathologies. Abdominal CT scan revealed presence of an infiltrating lesion that originated in the cervix. The $8 \times 5 \mathrm{~cm}$ lesion was closely adjacent to the posterior wall of the urinary bladder and to the anterior wall of the rectum. Numerous lymph nodes of up to $1.5 \mathrm{~cm}$ diameter were also found in the area of internal iliac vessels on the right, and in the area of the common iliac vessels. Cystoscopy and rectoscopy did not confirm involvement of the urinary bladder and rectum by the neoplastic process. The blood cell count and basic biochemistry were within normal values. In the period from 5 January 2004 to 10 February 2004, external irradiation of the pelvic area was performed, using a dose of 50 Gy in 25 fractions and of the para-aortic lymph nodes, giving $36 \mathrm{~Gy}$ in 20 fractions. Along with irradiation, the patient received 4 courses of cisplatin at the dose of $40 \mathrm{mg}$ per square meters per week. During irradiation, the patient developed diarrhea that needed administration of standard antidiarrheal drugs, drip hydration, and potassium supplementation, and also presented with leucopenia requiring administration of growth factors. Two weeks after completion of radiochemotherapy, the patient was readmitted to the Oncological Gynecology Department due to considerable weakness, strong pain within the foot and 
shank areas, lumbo-sacral spine, and difficulties in self-reliant mobility. Gynecological examination revealed considerable remission of the cancerous infiltration of the vaginal part. Neurological examination found limited mobility of the lumbar spine, coxalgia limiting the rotation movement in the hip joints, weakening of pain and touch sensations in respect to $L 4, L 5, S 1$ roots. The sonographic image of abdominal and smaller pelvis organs did not show any pathologies. X-ray examination of lumbosacral spine revealed osteoporosis. The patient started treatment with alendronic acid preparations. Due to anemia ( $\mathrm{Hb} 7.3$, Hct 20.8) the patient was given 2 units of blood group concordant red cell concentrate and iron preparations, which resulted in normalization of the hemoglobin level. When the patient's general condition improved, between 1 March 2004 and 3 April 2004, brachytherapy with a 40 Gy dose administered in 3 weekly fractions was performed. On gynecological examination after completion of brachytherapy, total clinical regression of the vaginal infiltration was observed. Over the period of 3 months following completion of oncological treatment, the patient reported recurrent abdominal pains, nausea, and vomiting, requiring several surgical consultations. The imaging examination (abdominal sonography) excluded obstruction and recurrence of tumor. Antibiotics and non-steroid anti-inflammatory drugs were used, and regression of symptoms was obtained. Mobility problems reported by the patient after completion of teletherapy and pain in the lumbosacral spine were slowly but steadily growing. Since October 2004, the patient has been under the orthopedic and neurological care of physicians in her local hospital. Neurological examination revealed paralysis of lower extremities, absence of deep reflexes, impaired pain and temperature sensation in the lower extremities. Symptomatic treatment involved steroids, analgesics including narcotics, neurological drugs such as galantamine hydrobromide preparations and alendronic acid preparations. The patient has been receiving physiotherapy. After six months of pharmacological therapy and intensive physiotherapy, neurological examination showed paralysis of the lower extremities, more intense on the left, persistent absence of deep reflexes and impaired pain and temperature sensation. The patient continued to complain of lumbosacral spine pain but started to ambulate with the aid of crutches. Her quality of life considerably deteriorated in April 2005, due to recto-vaginal fistula. An artificial anus was formed in the local hospital. Between 2005 and 2008, physiotherapy, pharmacological therapy and general strengthening therapy were carried out. MRI of the pelvis and thoracic spine was performed twice as well as $C T$ with bone algorithm. The examination performed in 2005 revealed the presence in the dural sac of hypointense areas with non-homogeneity of tracer uptake, the largest of them, $10 \times 6 \mathrm{~mm}$ in size, at the tracheal bifurcation level. The radiological image, along with the persistent neurological symptoms, raised suspicion of meningeal metastases. In the MRI scan performed in May 2008, a hypointense, non-homogeneous area with weak tracer uptake was found in the lateral sacral mass. CT with bone algorithm, performed in June 2008, revealed preserved outline of the sacral bone compact substance, non-homogeneous structure of lateral sacral mass, preserved sacral foramina outlines, radicular cysts within the sacral foramina, the iliac alas and L4, L5 vertebrae without any suspicious lesions. In the opinion of the radiologists, the image of the sacral bone in both examinations performed in 2008 was rather suggestive of reactive lesions. Bone scintigraphy showed only slightly increased tracer uptake in the area of the right sacroiliac joint which was considered nonspecific. Scans and periodical gynecological examinations did not show any cervical cancer recurrences. Further progressive, although small and slow improvement of the patient's neurological condition has been observed since 2006. At her last checkup, in April 2010, the patient ambulated self-sufficiently with a little aid of crutches, paralysis of the lower extremities was less strong, but absence of deep reflexes persisted and the pain and temperature sensation in the lower extremities continued to be impaired. The gynecological examination and MRI scan did not show any signs of cervical cancer recurrence. The artificial anus functions well. The patient has fully accepted this solution for the recto-vaginal fistula and does not, as yet, give consent to reconstruction of the continuity of the alimentary tract, though, in the future, she might change her mind.

\section{Discussion}

The paper presents a case of a cervical cancer patient successfully treated with irradiation and cisplatin, who developed serious radiotherapy-induced complications in the form of recto-vaginal fistula and lumbosacral plexopathy. While the incidence of this first complication ranges from $0.8 \%$ to $3.0 \%$, the radiotherapy-induced impairment of the lumbosacral plexus in cervical cancer patients treated with irradiation is very rare. The majority of post-irradiation reactions in the lower colon are manifested within 3 years after radiotherapy. The risk of the most serious complication in the colon, represented by recto-vaginal fistula, increases with the increase of the maximum total dose received from tele- and brachytherapy. With the total dose to the rectum of $80 \mathrm{~Gy}$, the risk of fistula is about $5 \%$. The remaining risk factors are the irradiation fraction dose, use of two treatment methods (surgery and irradiation) and individual predispositions. In the discussed case, the maximum total dose to the rectum was 73 Gy. Rare reports of radiation-induced damage to the lumbosacral plexus refer to irradiation of the small pelvis area with a dose generally exceeding 60 Gy $[1,2]$ or without including the para-aortic nodes [3]. The frequency of radiationinduced plexopathy ranges from $0.3 \%$ to $1.3 \%[4,5]$.

Acute, transient symptoms attributable to plexus lesion may also appear in the course of or soon after irradiation. Subacute, usually also transient, symptoms may appear within 6 months after radiotherapy of small pelvis tumors - tumors of the reproductive organ, testis, rectum, lymphomas etc. [6,7]. According to data from the literature, it may take even 30 years from the end of radiotherapy to occurrence of neurological symptoms [8-12]. Symptoms, in the form of strong, persistent pain, reduced muscular strength, and impairment of sensation in the lower extremities, recede over several weeks, but some of them are irreversible neurological problems, generally progressing, dramatically lowering the quality of life and largely impairing self-sufficient func- 
tioning of patients often cured from cancer $[1,3,13]$. In the case described by us, the first neurological symptoms, manifesting as pain in feet and shanks, lumbosacral spine, and difficulties in self-sufficient mobility, appeared a few weeks after completion of radiochemotherapy and progressed over the subsequent months, ending as clinical symptoms of paralysis of the lower extremities. Intensive physiotherapy, continued for over 6 years and still ongoing, has brought improvement of the neurological condition, allowing the patient to ambulate with crutches. Of fundamental importance is the systematic application of physical therapy, as even a short break causes rapid deterioration of our patient's motion efficiency. The significance of widely understood rehabilitation as well as pharmacological treatment is also stressed by other authors [13-15].

Georgius et al. [2] describe 4 cases of lumbosacral plexopathy in cervical cancer patients treated with irradiation. In three of them, the dominating symptom was impairment of muscular strength in the lower extremities, while in one patient it was progression of neurological lesions causing paralysis of the lower extremities. Some patients were also reported to have urinary and fecal incontinence $[1,16]$. There are reports that point to stabilization or progression of neurological symptoms over a few months or a few years $[11,17,18]$. One of the rare cases of improvement of the patient's neurological condition is described by Coulombe [3] and is similar to the situation presented by us.

Apart from detailed neurological examination and history (previous irradiation, absence of earlier neuropathy symptoms), it may be useful to perform electromyography, cerebrospinal fluid analysis as well as CT, MRI and PET [1-3,13,19]. Imaging examinations allow one, first of all, to exclude tumor involvement of the nervous system, while they are not always helpful in the assessment of radiotherapy-induced plexopathy.

The mechanism of post-irradiation lesion of the lumbosacral plexus is not quite clear. The effect of tissue irradiation depends on total dose, fraction dose, irradiation technique applied, concomitant chemotherapy, and intraoperative intracavitary brachytherapy. Local ischaemia and fibrosis of soft tissues may be associated with microcirculatory deficiency. Administration of a dose exceeding 10 Gy has caused changes in Schwann cells, endoneural fibroblasts, small vessel wall cells and in perineural cells [20]. Clinical symptoms of plexopathy were observed both when doses for the plexus were below 60 Gy and when they were higher [21]. Coulombe [3] draws attention to the use of cisplatin in radiochemotherapy.

Although the risk of damage to the lumbosacral plexus in the course of radiotherapy in tumors of the reproductive organ is very low, the example described here as well as the cited items from the literature show that it presents one of the most serious complications induced by radiation.

\section{References}

1. Lee SS, Lee SH. Radiation lumbosacral plexopathy in a patient of cervical cancer with long-term follow-up. J Korean Neurol Assoc 2005; 23: 436-7.
2. Georgiou A, Grigsby PW, Perez CA. Radiation induced lumbosacral plexopathy in gynaecologic tumors: clinical findings and dosimetric analysis. Int J Radiat Oncol Biol Phys 1993; 26: 479-82.

3. Coulombe G, Thiessen B, Balkwill S, Aquino-Parsons C. Polyradiculopathy post-concomitant chemoradiation for carcinoma of the uterine cervix treated with pelvic and para-aortic fields. Gynecol Oncol 2005; 99: 774-7.

4. Ashenhurst EM, Quartey GR, Starreveld A. Lumbo-sacral radiculopathy induced by radiation. Can J Neurol Sci 1977; 4: 259-63.

5. Yadav RR. Radiation-induced lumbosacral plexopathy. http:// www.emedicine.com./pmr/topic90.htm.

6. Frykholm GJ, Sintorn K, Montelius A, Jung B, Påhlman L, Glimelius B. Acute lumbosacral plexopathy during and after preoperative radiotherapy of rectal adenocarcinoma. Radiother Oncol 1996; 38: 121-30.

7. Brydřy M, Storstein A, Dahl O. Transient neurological adverse effects following low dose radiation therapy for early stage testicular seminoma. Radiother Oncol 2007; 82: 137-44.

8. Stryker JA, Sommerville K, Perez R, Velkley DE. Sacral plexus injury after radiotherapy for carcinoma of the cervix. Cancer 1990; 66: 1488-92.

9. Gonzales-Cabalero G. Lumbosacral plexopathy 15 years after radiotherapy for carcinoma of the cervix. Rev Neurol 2000; 30: 97 (Abstract).

10. Thomas JE, Cascino TL, Earle JD. Differential diagnosis between radiation and tumor plexopathy of the pelvis. Neurology 1985; 35: 1-7.

11. Pettigrew LC, Glass JP, Maor M, Zornoza J. Diagnosis and treatment of lumbosacral plexopathies in patients with cancer. Arch Neurol 1984; 41: $1282-5$

12. Numata K, Ito M, Uchiyama S, Kobayashi I, Takemiya T, Maruyama S. A case of delayed radiation lumbo-sacral plexopathy. No To Shinkei 1990; 42: 629-33.

13. Abu-Rustum NR, Rajbhandari D, Glusman S, Massad LS. Acute low er extremity paralysis following radiation therapy for cervical cancer. Gynecol Oncol 1999; 75: 152-4

14. Topikan E, Onal HC, Yavuz AA, Yavuz MN. Pathophysiology and management of radiation-induced lumbosacral plexopathy. Turk Onkoloji Dergisi 2008; 23: 147-52.

15. Ozçakar L, Kara M, Erol O, Kaymak B, Akinci A, Ta kiran C, Ayhan A A reappraisal on the management of lumbosacral plexopaties in gynaecological malignancies: where do the physiatrists stand? Int J Gynecol Cancer 2005; 15: 915-7.

16. Iglicki F, Coffin B, Ille O, Flourié B, Amarenco G, Lémann M, Messing $B$. Fecal incontinence after pelvic radiotherapy: evidences for a lumbosacral plexopathy. Report of a case. Dis Colon Rectum 1996; 39 : 465-7.

17. Jaeckle KA. Neurological manifestations of neoplastic and radiationinduced plexopathies. Semin Neurol 2004; 24: 385-93.

18. Jaeckle KA, Young DF, Foley KM. The natural history of lumbosacral plexopathy in cancer. Neurology 1985; 35: 8-15.

19. Mammen AL, Chaudhry V. The difficulties of diagnosis neuromuscular complications. J Support Oncol 2005; 3: 285-6.

20. Bradley WG, Fewings JD, Cumming WJ, Harrison RM. Delayed myeloradiculopathy produced by spinal X-irradiation in the rat. J Neurol Sci 1977; 31: 63-82.

21. Aho K, Sainio K. Late irradiation-induced lesions of the lumbosacral plexus. Neurology 1983; 33: 953-5.

\section{Address for correspondence}

\section{Matgorzata Klimek}

Klinika Ginekologii Onkologiczne

Centrum Onkologii - Instytut im. Marii Skłodowskiej-Curie

ul. Garncarska 11

31-115 Kraków

e-mail: klimekmag@poczta.onet.pl

$\begin{array}{ll}\text { Submitted: } & 15.01 .2011 \\ \text { Accepted: } & 18.01 .2012\end{array}$ 